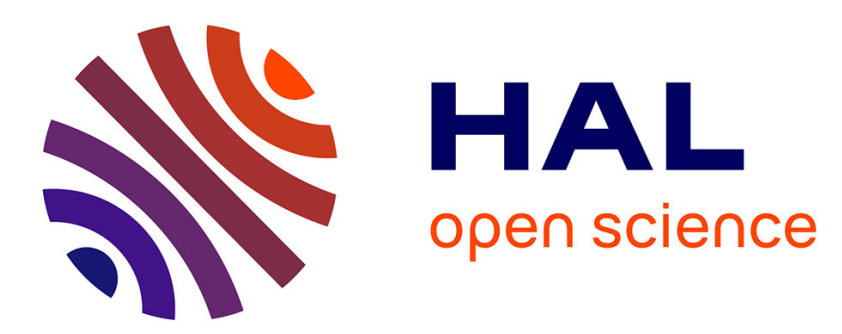

\title{
Simulating diffusion processes in discontinuous media: a numerical scheme with constant time steps
}

Antoine Lejay, Géraldine Pichot

\section{To cite this version:}

Antoine Lejay, Géraldine Pichot. Simulating diffusion processes in discontinuous media: a numerical scheme with constant time steps. Journal of Computational Physics, 2012, 231 (21), pp.7299-7314. 10.1016/j.jcp.2012.07.011 . hal-00649170v3

\section{HAL Id: hal-00649170 \\ https://hal.inria.fr/hal-00649170v3}

Submitted on 20 Aug 2012

HAL is a multi-disciplinary open access archive for the deposit and dissemination of scientific research documents, whether they are published or not. The documents may come from teaching and research institutions in France or abroad, or from public or private research centers.
L'archive ouverte pluridisciplinaire HAL, est destinée au dépôt et à la diffusion de documents scientifiques de niveau recherche, publiés ou non, émanant des établissements d'enseignement et de recherche français ou étrangers, des laboratoires publics ou privés. 


\title{
Simulating diffusion processes in discontinuous media: a numerical scheme with constant time steps
}

\author{
Antoine Lejay ${ }^{1,2,3,4,5} \quad$ Géraldine Pichot ${ }^{6,7}$
}

August 20, 2012

\begin{abstract}
In this article, we propose new Monte Carlo techniques for moving a diffusive particle in a discontinuous media. In this framework, we characterize the stochastic process that governs the positions of the particle. The key tool is the reduction of the process to a Skew Brownian Motion (SBM). In a zone where the coefficients are locally constant on each side of the discontinuity, the new position of the particle after a constant time step is sampled from the exact distribution of the SBM process at the considered time. To do so, we propose two different but equivalent algorithms: a two-steps simulation with a stop at the discontinuity and a one-step direct simulation of the SBM dynamic. Some benchmark tests illustrate their effectiveness.
\end{abstract}

Keywords: divergence form operators, stochastic differential equation, skew Brownian motion, Monte Carlo simulation, Euler scheme, geophysics, diffusive media with interfaces.

\footnotetext{
${ }^{1}$ Université de Lorraine, IECN, UMR 7502, Vandœuvre-lès-Nancy, F-54500, France

${ }^{2}$ CNRS, IECN, UMR 7502, Vandœuvre-lès-Nancy, F-54500, France

${ }^{3}$ Inria, Villers-lès-Nancy, F-54600, France

${ }^{4}$ Contact: IECN, BP 70238, F-54506 Vandouvre-lès-Nancy CEDEX, France. Email:

Antoine.Lejay@iecn.u-nancy.fr

${ }^{5}$ This work has been supported by the ANR SIMUDMRI (ANR-10-COSI-SIMUDMRI).

${ }^{6}$ Inria, Rennes, France

${ }^{7}$ Contact: INRIA, Campus de Beaulieu, 35042 Rennes Cedex, France. Email: Geraldine.Pichot@inria.fr
} 


\section{Introduction}

In many domains of physics and biology, one faces parabolic partial differential equations of type

$$
\frac{\partial C(t, x)}{\partial t}=\frac{1}{2} \nabla(D(x) \nabla C(t, x))
$$

where $C$ represents for example the concentration or the density of a population, and $D$ is a diffusivity tensor. This equation arises naturally as it relates the variation of $C(t, x)$ in time to the divergence of the flux (case of an incompressible fluid) in a media where the Fick law occurs (the flux $J$ is related to the gradient of the concentration through $\left.J=\frac{1}{2} D \nabla C\right)$. This is for example the case of a fluid in a porous medium and (1) is then the Darcy law.

Equations of type (1) may be naturally solved by a Monte Carlo method by relating the value of $C(t, x)$ with the density of particles moving randomly in the medium with a dynamic prescribed by the second-order differential operator $\nabla(D \nabla \cdot)$. If $D$ is smooth, then the particles move as the solutions to some Stochastic Differential Equation (SDE) $X_{t}=x+\int_{0}^{t} B\left(X_{s}\right) d W_{s}+$ $\int_{0}^{t} \nabla D\left(X_{s}\right) d s$, where the matrix $B$ is such that $B B^{T}=D$ and $W$ is a Brownian motion.

There are many situations where $D$ is discontinuous, and the discontinuity may have several order of magnitude. Even if $D$ is not smooth, a stochastic process $X$ is associated to $\frac{1}{2} \nabla(D \nabla \cdot)[60]$.

The simulation techniques we present here rely on the properties of $X$ in a one-dimensional random media. As explained in Remark 3, they may be applied as well as the solution to the Stochastic Differential Equation (SDE) $X_{t}=x+\int_{0}^{t} \sqrt{D\left(X_{s}\right)} d W_{s}$ for a Brownian motion $W$, when $D$ is discontinuous. The process is associated to the non-divergence form operator $\frac{1}{2} D \triangle$.

Monte Carlo methods are sometimes the only available methods and have the advantage to be simple to implement in general and may be combined with other effects such as physical or chemical interactions with the medium, ...

Discontinuities of $D$ happen in many scientific domains, where the question of the simulation of $X$ has been treated: geophysics $[1-3,5,11,17,27$, 31-33, 41, 43, 54-56,59,62], ecology [13,53,57], brain imaging [22], astrophysics [42,64], meteorology [61], molecular dynamic [9, 47], ...

As backed by numerical and experimental results [7], specific schemes have to be designed to take the discontinuities into account.

Although the multi-dimensional case remains open, the one-dimensional case is tractable and already arouses a lot of interest. Several approaches 
have already been proposed: in addition to the references given above, see [19-21,37, 39, 44, 45], ...

In this article, we propose a new simulation method relying on exact simulation on a simple one-dimensional case.

More precisely, we consider the divergence form operator

$$
L=\frac{1}{2} \nabla(D \nabla \cdot)
$$

in dimension one (note the one-half), where $D(x)=D^{+}$if $x \geq 0$ and $D(x)=$ $D^{-}$if $x<0$. We want to construct a numerical scheme for the stochastic process $X$ associated to $L$, where $X$ evolves according to constant time steps.

By comparison with the Continuous Time Random Walk (CTRW) approach, the time steps are chosen here as constants while in the CTRW framework they are random $[43,48,49,52]$. The advantage of CTRW is to be able to deal with normal or anomalous diffusion. Despite being restricted to normal diffusion, our algorithms present the major interest of simulating the exact distribution of the SBM process.

As explained below, considering $D$ piecewise constant with a discontinuity at 0 is the simplest case but one may of course consider the case of a diffusion coefficient $D$ which is not piecewise constant or with multiple discontinuities, since the dynamic of the particle depends mainly on its immediate environment. This way, our scheme has to be seen as a "basic brick" that is to be applied only locally around a discontinuity.

For this, we rely on a change of variable that transforms $X$ into a Skew Brownian motion (SBM) $[26,28,38]$. This change of variable has already been used in several articles $[19,37,39,54-56]$ and the SBM is now acknowledged to be one of the main tool to understand the behavior of the process around a discontinuity of $D$. This probabilistic reasoning gives then a simple, analytic expression for the transition density function $\mathfrak{q}(t, x, y)$ of $X$, which is the density of $X_{t}$ when $X_{0}=x$ is known. This expression for the density may also be obtained by the method of images [31,62].

Other numerical schemes relying on the SBM have already been proposed. Some of them are not schemes with constant time steps $[20,37,39]$. In [19], P. Étoré proposes a way to move the particle on a fixed grid. The Euler scheme proposed by M. Martinez and D. Talay [44-46] relies on another transform and is different from ours. In [16], the authors use this kind of transform together with the Girsanov transform to study the density of the process with a drift and discontinuous coefficients. In [21], P. Étoré and M. Martinez proposed a scheme with a constant time step which is a variation of the exact simulation method of A. Beskos and G.O Roberts [8]. With 
respect to ours, this method may be applied to the case of a variable diffusion coefficient with a drift. But it is more complex to set up.

The novelty of this article is to explain how to generate efficiently a random variate with density $\mathfrak{q}(t, x, \cdot)$. Note that our algorithms are exact and do not introduce discretization errors. Generating such a random variate allows one to draw the position $X_{(k+1) \delta t}$ of the particle when its position $X_{k \delta t}$ at time $k \delta t$ is known. This way, an approximation of the path of the particle may be constructed and we call this an Euler scheme, although it is different in essence from the Euler scheme for solutions to SDE.

Te paper is organized as follows. Sect. 2 presents the Euler scheme and introduces the SBM process. A recall of the dynamic of the SBM is done in Sect. 3. Then we present the main contributions of this paper which are two different but equivalent algorithms to simulate the SBM dynamic. The first one, presented in Sect. 4, relies on the computation of the first passage at the interface which is modelled by a discontinuity. The reason is that once at the interface, the simulation of the position of the particle at a given time is easily deduced from the one of a normal random variable. The second algorithm, presented in Sect. 5, relies on a direct simulation of the random variable by using the special form of the density. Sect. 6 illustrates the effectiveness of the proposed algorithms on some benchmark tests.

Notations and definitions A random variate is a realization of a random variable. In this article, every time an algorithm relies on the generation of several random variates, they are assumed to be independent. In practice, we use only a pseudo-random number generator.

We denote by $\mathcal{N}(\mu, t)$ the Gaussian distribution with mean $\mu$ and variance $t$. The Gaussian density is

$$
\mathfrak{g}(t, x)=\frac{1}{\sqrt{2 \pi t}} \exp \left(\frac{-x^{2}}{2 t}\right)
$$

and we set $\mathfrak{g}(x)=\mathfrak{g}(1, x)$.

The cumulative distribution function of the normal distribution $\mathcal{N}(0,1)$ is

$$
\Phi(x)=\mathbb{P}[G \leq x]=\int_{-\infty}^{x} \frac{1}{\sqrt{2 \pi}} \exp \left(\frac{-y^{2}}{2}\right) d y=\int_{-\infty}^{x} \mathfrak{g}(y) d y,
$$

and with set

$$
\bar{\Phi}(x)=1-\Phi(x)=\mathbb{P}[G>x]
$$

with $G \sim \mathcal{N}(0,1)$.

The uniform distribution on an interval $[a, b)$ is denoted by $\mathcal{U}([a, b))$. The Bernoulli distribution of parameter $p$ is denoted by $\operatorname{Ber}(p)$. 
A Brownian motion $B$ with $B_{0}=x$ is simulated very easily by iteratively computing $B_{t+\delta t}=B_{t}+\xi$ where $\xi$ is a realization of $\mathcal{N}(0, \delta t)$ and is independent from $B_{t}$.

\section{The Skew Brownian motion and the Euler scheme}

We consider the divergence form operator

$$
L=\frac{1}{2} \nabla(D \nabla \cdot)
$$

in dimension one (note the one-half), where $D(x)=D^{+}$if $x \geq 0$ and $D(x)=$ $D^{-}$if $x<0$.

Following standard results in PDE (See [35] for example), for a square integrable function $f$ (resp. $g$ ), solutions of the elliptic (resp. parabolic) PDE

$$
\left\{\begin{array} { l } 
{ L u = f \text { on } ( - \ell _ { 1 } , \ell _ { 2 } ) , } \\
{ u ( - \ell _ { 1 } ) = \alpha , } \\
{ u ( \ell _ { 2 } ) = \beta }
\end{array} \quad \left(\operatorname{resp} . \quad\left\{\begin{array}{l}
\left.\partial_{t} v(t, x)=L v(t, x), t>0, x \in \mathbb{R}, \quad\right) \\
v(0, x)=g(x)
\end{array}\right.\right.\right.
$$

is smooth on $\left(-\ell_{1}, \ell_{2}\right) \backslash\{0\}$ (resp. $\left.\mathbb{R}_{+} \times(\mathbb{R} \backslash\{0\})\right)$, and the conditions

$$
\begin{aligned}
& u(0+)=u(0-) \text { and } D^{+} \nabla u(0+)=D^{-} \nabla u(0-) \\
& \left(\text { resp. } u(t, 0+)=u(t, 0-) \text { and } D^{+} \nabla u(t, 0+)=D^{-} \nabla u(t, 0-), \forall t>0\right) .
\end{aligned}
$$

The condition on the gradients in (2) is called the flux condition.

Remark 1 . More generally, we may consider a problem with diffusivity $D^{+}$on the right side of $0, D^{-}$on the left side of 0 and a solution which is continuous at 0 with a flux condition $(1+q) \nabla u(0+)=(1-q) \nabla u(0-), q \in(-1,1)$. For this, select four values $\left(\bar{D}^{+}, \bar{D}^{-}, \underline{D}^{+}, \underline{D}^{-}\right)$such that

$$
\underline{D}^{-}=1, q=\frac{\bar{D}^{+}-\bar{D}^{-}}{\bar{D}^{+}+\bar{D}^{-}}, D^{+}=\frac{\bar{D}^{+}}{\underline{D}^{+}} \text {and } D^{-}=\frac{\bar{D}^{-}}{\underline{D}^{-}}
$$

and use the operator $L=\frac{1}{2 \underline{D}} \nabla(\bar{D} \nabla \cdot)$. See [39] for details.

Following [43], $\bar{D}$ may be interpreted as a diffusivity factor (in meters) while $1 / \underline{D}$ may be interpreted as a velocity called the parametric velocity (in $\mathrm{m} / \mathrm{s}$ ). Thus $D=\bar{D} / 2 \underline{D}$ is the diffusivity coefficient (in $\mathrm{m}^{2} / \mathrm{s}$ ), while $\bar{D} \underline{D}$ (in s) has the dimension of a time and is used as the mean time of the time jumps in the Continuous Time Random Walk technique.

For the sake of simplicity, we consider only divergence form operators in this article but everything could be carried to general framework as shown in Remarks 3 and 4 below. 
Our aim is to design a numerical scheme for the stochastic process $X$ associated to $L$ with a constant time step. This means that for a given time step $\delta t$ and a given position $X_{t}$ of the particle, how to simulate the next position $X_{t+\delta t}$ ?

Let us recall that the process $X$ is in some sense "memoryless", which is rigorously expressed by the Markov property. This means that the future positions of $\left(X_{s}\right)_{s \geq t}$ when $X_{t}$ is known depend only on $X_{t}$ and not on $\left(X_{r}\right)_{r<t}$. Besides, these processes are homogeneous in time, which means that the distribution of $\left(X_{t+\theta}\right)_{\theta \geq 0}$ given $X_{t}=x$ is the same as the distribution of $\left(X_{\theta}\right)_{\theta \geq 0}$ given $X_{0}=x$. These two properties are pretty convenient for writing numerical schemes with constant time steps: one has only to prescribe, when $X_{0}=x$, how to find exactly or approximately the position $y$ of the particle at time $X_{\delta t}$, and then to start again by replacing $x$ by $y$.

We will abusively called a constant time step scheme an Euler scheme, by similarity with the most common and widely used Euler scheme for solving SDE, although our process $X$ belongs to a more general class of stochastic processes.

For convenience, we have placed the discontinuity at $x_{d}=0$. In the case of diffusivity coefficient $D$ discontinuous at some point $x_{d} \neq 0$, it is sufficient to shift the old and new positions respectively by $-x_{d}$ and $x_{d}$ for reducing this case to $x_{d}=0$.

Remark 2. According to the respective values of $D^{+}$and $D^{-}$, it may be convenient to use different time steps when the particle is on the right or on the left of the discontinuity. As we deal here with the dynamic of the particle close to the discontinuity, we do not address this problem here.

\subsection{Reduction to the Skew Brownian motion}

It has been proved that the process $X$ is solution to a stochastic differential equation (SDE) with local time (See $[19,39]$ for example):

$$
X_{t}=x+\int_{0}^{t} \sqrt{D\left(X_{s}\right)} d W_{s}+\gamma L_{t}^{0}(X), \gamma=\frac{D^{+}-D^{-}}{D^{+}+D^{-}} .
$$

Here, $L_{t}^{0}(X)$ is the symmetric local time of $X$. This is a particular process which describes the amount of time the process spends to 0. It may be formally defined as

$$
L_{t}^{0}(X)=\lim _{\epsilon \rightarrow 0} \frac{1}{2 \epsilon} \int_{0}^{t} \mathbf{1}_{\left\{X_{s} \in[-\epsilon, \epsilon]\right\}} d\langle X\rangle_{s},
$$

where $\langle X\rangle$ is the quadratic variation of $X$, which is $\langle X\rangle_{t}=\int_{0}^{t} D\left(X_{s}\right) d s$. This is a continuous, non-decreasing process. However, it increases only on the closure of the set $\left\{t \geq 0 \mid X_{t}=0\right\}$, which is a set of zero Lebesgue's measure. 
With $\Psi(x)=x / \sqrt{D^{+}}$if $x \geq 0$ and $\Psi(x)=x / \sqrt{D^{-}}$if $x<0$, the ItôTanaka formula [30, Theorem 3.7.1] and the properties of the local time yields that $Y_{t}=\Phi\left(X_{t}\right)$ is solution to

$$
Y_{t}=\Psi(x)+W_{t}+\theta L_{t}^{0}(Y), \theta=\frac{\sqrt{D^{+}}-\sqrt{D^{-}}}{\sqrt{D^{+}}+\sqrt{D^{-}}} .
$$

The process $Y$ is called the skew Brownian motion (SBM) of parameter $\theta$. The survey article [38] contains an account about the possible constructions and the main properties of this process which was introduced in the 70's.

The change of variable $\Psi$ has been used in [37,39] and in [54-56].

The density transition function $\mathfrak{q}(t, x, y)$ of the process $X$ is linked to the density transition function $\mathfrak{p}_{\theta}$ of the $\mathrm{SBM}$ of parameter $\theta$ given by (3) by

$$
\mathfrak{q}(t, x, y)=\frac{1}{\sqrt{D(y)}} \mathfrak{p}_{\theta}\left(t, \frac{x}{\sqrt{D(x)}}, \frac{y}{\sqrt{D(y)}}\right)
$$

A simple, analytic expression of $\mathfrak{p}_{\theta}$ is given by (5) below.

The meaning of the function $\mathfrak{q}(t, x, y)$ is that when being at $x$ at time 0 , the probability that the particle moving according to $L$ with a discontinuity at 0 belongs to the infinitesimal interval of size $d y$ around $y$ is $\mathfrak{q}(t, x, y) d y$.

This change of variable is pretty simple and may be applied only locally. Algorithm 1 below means that when the particle $X$ is close enough to the boundary (See Sect. 2.2), we first transform it into a Skew Brownian motion by $Y_{t}=X_{t} / \sqrt{D\left(X_{t}\right)}$. Second, we apply one of the schemes given below to simulate $Y_{t+\delta t}$. Third, we find the position of the particle at time $t+\delta t$ by $X_{t+\delta t}=\sqrt{D\left(Y_{t+\delta t}\right)} Y_{t+\delta t}$.

Remark 3. Similar computations may be applied to the solution of the SDE $X_{t}=x+\int_{0}^{t} \sqrt{D\left(X_{s}\right)} d B_{s}$ whose infinitesimal generator is $\frac{1}{2} D \triangle$. More generally, as noted in Remark 1, we may consider the process $X$ generated by $L=\frac{1}{2 D} \nabla(\bar{D} \nabla \cdot)$. In this case, define $\Phi(x)=\beta^{+} x$ for $x \geq 0$ and $\Phi(x)=\beta^{-} x$ with $\beta^{ \pm}=\sqrt{\underline{D}^{ \pm}} / \sqrt{\bar{D}^{ \pm}}$. Hence $\Phi(X)$ is a SBM with

$$
\theta=\frac{\sqrt{\bar{D}^{+} \underline{D}^{+}}-\sqrt{\bar{D}^{-} \underline{D}^{-}}}{\sqrt{\bar{D}^{+} \underline{D}^{+}}+\sqrt{\bar{D}^{-} \underline{D}^{-}}}
$$

as seen in $[19,39]$. In particular, the SBM of parameter $\theta$ may be associated to $\bar{D}^{+}=\underline{D}^{+}=(1+\theta) /(1-\theta)$ and $\bar{D}^{-}=\underline{D}^{-}=1[38]$. 
Remark 4 . The density transition function $\mathfrak{q}(t, x, y)$ of the process $X$ generator by $L=\frac{1}{2 \underline{D}} \nabla(\bar{D} \nabla \cdot)$ satisfies both the Kolmogorov backward equation

$$
\frac{\partial \mathfrak{q}(t, x, y)}{\partial t}+\frac{1}{2 \underline{D}(x)} \nabla_{x}\left(\bar{D}(x) \nabla_{x} \mathfrak{q}(t, x, y)\right)=0
$$

as a function of $x$ and the Kolmogorov forward equation

$$
\frac{\partial \mathfrak{q}(t, x, y)}{\partial t}=\frac{1}{2} \nabla_{y}\left(\bar{D}(y) \nabla_{y}\left(\frac{1}{\underline{D}(y)} \mathfrak{q}(t, x, y)\right)\right)
$$

as a function of $y$. This expresses that at 0

$$
\mathfrak{q}(t, 0+, y)=\mathfrak{q}(t, 0-, y) \text { and } \bar{D}^{+} \nabla_{x} \mathfrak{q}(t, 0+, y)=\bar{D}^{+} \nabla_{x} \mathfrak{q}(t, 0-, y)
$$

for any $y$, while

$$
\frac{1}{\underline{D}^{+}} \mathfrak{q}(t, x, 0+)=\frac{1}{\underline{D}^{-}} \mathfrak{q}(t, x, 0-) \text { and } \frac{\bar{D}^{+}}{\underline{D}^{+}} \nabla_{y} \mathfrak{q}(t, x, 0+)=\frac{\bar{D}^{-}}{\underline{D}^{-}} \nabla_{y} \mathfrak{q}(t, x, 0-)
$$

for any $x$. As noted in the end of Remark 3, the SBM of parameter $\theta$ belongs to this class of processes. Thus, the first condition in (4) becomes (1$\theta) \mathfrak{p}_{\theta}(t, x, 0+)=(1+\theta) \mathfrak{p}_{\theta}(t, x, 0-)$ and explains the discontinuities observed in Figure 2.

\subsection{The interface layer}

The SBM behaves like a Brownian motion except when it reaches 0 . Let $\tau$ be the first time at which the Brownian motion starting from $x<0$ reaches 0 . Then $\mathbb{P}_{x}[\tau<t]=2 \Phi(x / \sqrt{t})$ when $x<0$. Fix a probability $\alpha \in(0,1)$ and find $c_{\alpha}>0$ such that $2 \Phi\left(-c_{\alpha}\right)=\alpha$. Then with probability $\alpha$, the SBM does not reach the interface at position 0 for $x<-c_{\alpha} \sqrt{t}$. For $\alpha=5 \%, c_{\alpha}=1.95$. For $\alpha=1 \%, c_{\alpha}=2.57$. For $\alpha=0.5 \%, c_{\alpha}=2.80$.

As summarized in Figure 1, the idea is then to use the usual Euler scheme with step $\delta t$ for the Brownian motion when the particle does not belong to $\left[-\sqrt{D^{-}} c_{\alpha} \sqrt{\delta t}, \sqrt{D^{+}} c_{\alpha} \sqrt{\delta t}\right]$, and a biased Euler scheme with step $\delta t$ when the particle belongs to this interval $\left[-\sqrt{D^{-}} c_{\alpha} \sqrt{\delta t}, \sqrt{D^{+}} c_{\alpha} \sqrt{\delta t}\right]$, which we call the interface layer. By a biased Euler scheme, we mean a scheme that replicates the dynamic of the SBM.

\subsection{The Euler scheme}

A scheme for $X$ will be deduced from a scheme on $Y$ by simply applying the function $\Psi^{-1}$ to the next position of the particle. Away from the interface, the usual Euler scheme (or any other efficient scheme) may be used whereas 


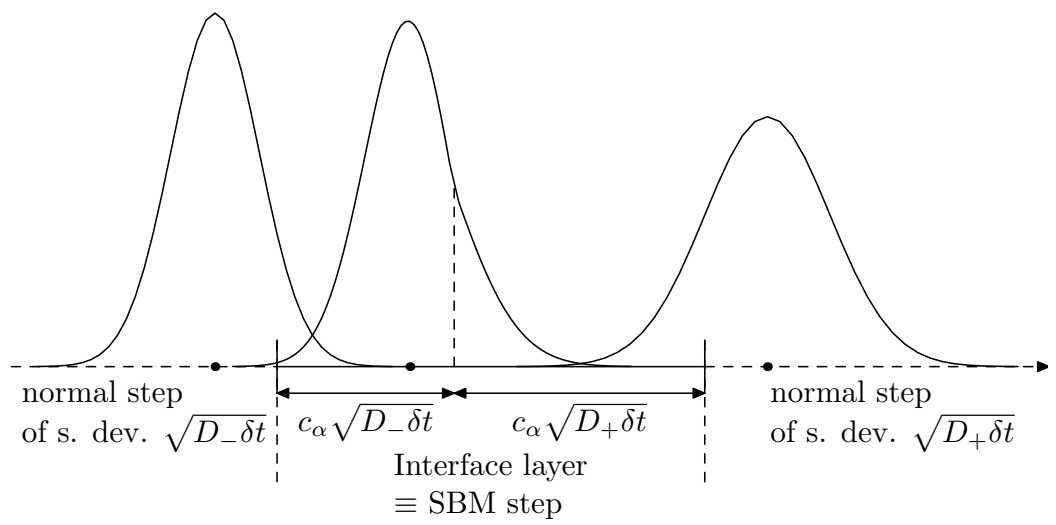

Figure 1: The interface layer $\left[-\sqrt{D^{-}} c_{\alpha} \sqrt{\delta t}, \sqrt{D^{+}} c_{\alpha} \sqrt{\delta t}\right]$; Outside the interface layer: normal step; Inside the interface layer: SBM step (notice the non Gaussian character of the corresponding density).

inside the interface, a biased scheme is proposed: See Algorithm 1. Also, there is no problem in dealing with multiple interfaces.

We now concentrate on the simulation of the biased Euler scheme that gives the next position of a particle starting within the interface layer.

\section{The dynamic of the Skew Brownian motion}

We have to focus on an approximation of the Skew Brownian motion at a given time $t$. This process has the Markov properties and is homogeneous in time, which means that the distribution of $\left(Y_{s+t}\right)_{t \geq 0}$ given $\left(Y_{r}\right)_{0 \leq r \leq s}$ has the same distribution as $\left(Y_{t}\right)_{t \geq 0}$ given $Y_{0}$.

Let $\mathbb{P}_{x}$ be the distribution of $Y$ with $Y_{0}=x$. With an abuse of notation, we use the same convention for the Brownian motion $W$.

The density transition function of the SBM for a discontinuity at $x_{d}=0$ has a simple expression:

$$
\mathfrak{p}_{\theta}(t, x, y)=\mathfrak{g}(t, y-x)+\operatorname{sgn}(y) \theta \mathfrak{g}(t,|y|+|x|)
$$

with $\operatorname{sgn}(x)=1$ if $x \geq 0$ and $\operatorname{sgn}(x)=-1$ if $x<0$. This expression may be derived from the reflection principle $[38,63]$. Figure 2 shows typical plots of the density.

More generally, for a discontinuity at $x_{d}$, the density transition function writes $\mathfrak{p}_{\theta, x_{d}}(t, x, y)=\mathfrak{p}_{\theta}\left(t, x-x_{d}, y-x_{d}\right)$.

From (5), we deduce immediately that the SBM enjoys the same scaling property as the Brownian motion. 


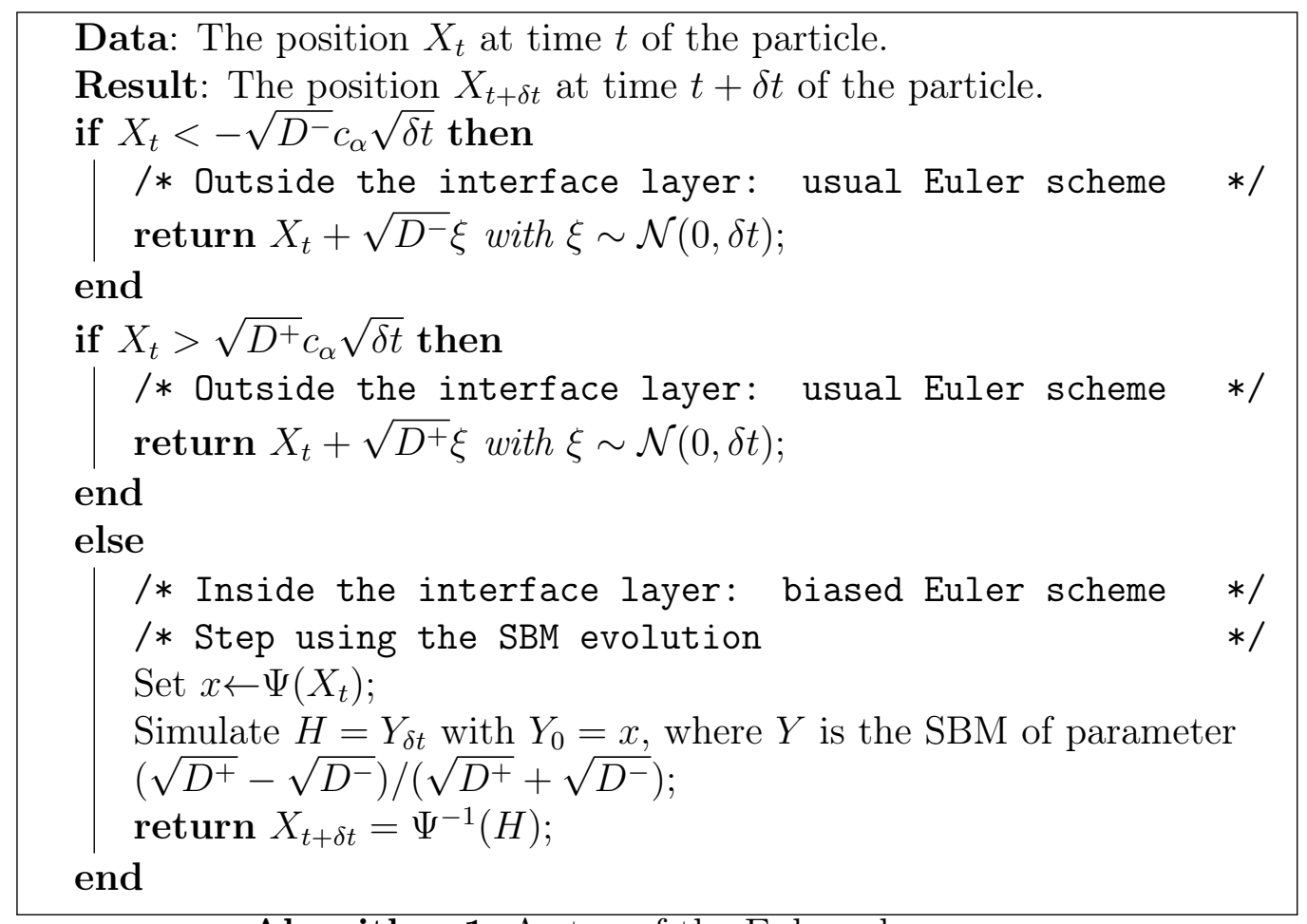

Algorithm 1: A step of the Euler scheme.

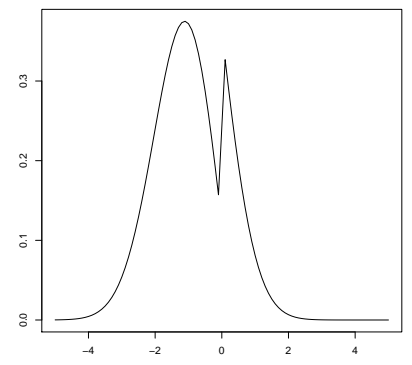

(a) $x=-1$

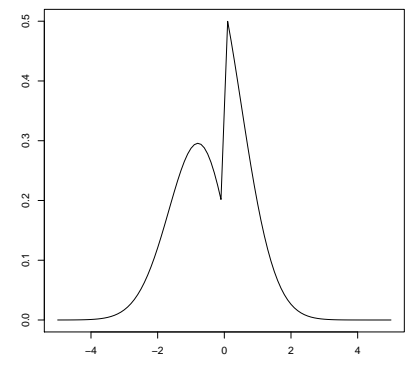

(b) $x=-0.5$

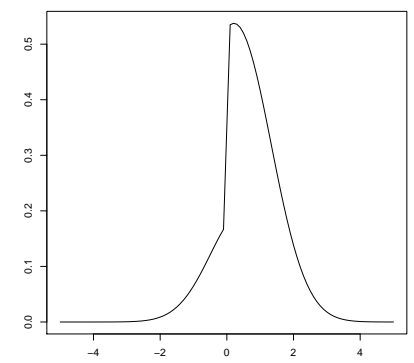

(c) $x=0.5$

Figure 2: The density $\mathfrak{p}_{\theta}(t, x, \cdot)$ of the Skew Brownian motion for $\theta=0.5$ at time $t=1$ for three values of the initial point $x$. 
Property 1 (Scaling property). For any $c>0$ and any $t>0, Y_{t}^{\prime}=\sqrt{c} Y_{t / c}$ with $Y_{0}^{\prime}=\sqrt{c} x$ is equal in distribution to $Y_{t}$ with $Y_{0}=x$.

The "symmetry" property is also useful and follows from the equality $\mathfrak{p}_{\theta}(t, x, y)=\mathfrak{p}_{-\theta}(t,-x, y)$. Note that $\theta$ is transformed into $-\theta$.

Property 2 ("Symmetry" property). For a SBM process $Y$ of parameter $\theta$ with $Y_{0}=x$, the process $Y^{\prime}=-Y$ is a $S B M$ of parameter $-\theta$ with $Y_{0}^{\prime}=-x$.

Under $\mathbb{P}_{x},|Y|$ has the same distribution as a reflected Brownian motion $|W|$. This is an immediate consequence of the Itô-Tanaka formula applied to $|\cdot|$. Besides $\mathbb{P}_{0}\left[Y_{t}>0\right]=(1+\theta) / 2$.

We recover the experimental result of [59]: The average number of particles at time $t$ that are on the right of the discontinuity is equal to $\sqrt{D^{+}} /\left(\sqrt{D^{+}}+\right.$ $\sqrt{D^{-}}$) time the number of particles.

The next two sections present the two algorithms we propose for the simulation of the dynamic of the SBM. The first one is a two-steps algorithm that contains a stop at the interface of discontinuity, the second one yields a direct simulation of the SBM process according to its known density transition function (5). An alternative but more complex algorithm, that relies on some fine properties of Brownian paths, may be found in [34].

\section{A two-steps algorithm for the simulation of the SBM evolution}

This algorithm requires to simulate the first time $\tau$ the process reaches zero, if it happens before $\delta t$. This aspect is developed in B. In particular, we present some existing methods that allow one to simulate the exact time it takes for the particle to reach the interface $[4,10,23,40]$. We also discuss the choice of a linear interpolation.

Starting at a position say $x<0$, once the time $\tau=\inf \left\{t>0 \mid W_{t}=0\right\}$ is known for a Brownian motion $W$ with $W_{0}=x$, one faces two options: either $\tau$ is greater than $\delta t$, which means the particle did not cross the interface of discontinuity during the time $\delta t$, either it is lower, then the particle is moved at the interface and one has to simulate its next position, during the remaining time $\delta t-\tau$.

In this last case, the starting point $x$ is then 0 , and $Y_{t}=\epsilon|G|$, where $G \sim \mathcal{N}(0, t)$, that is a normal of mean 0 and variance $t$, and $\epsilon \in\{-1,1\}$ is independent from $G$ and $\mathbb{P}[\epsilon=1]=(1+\theta) / 2$. This follows immediately from the symmetry property of the Gaussian distribution and the expression (5).

The SBM evolution step of Algorithm 1 is given by Algorithm 2 . 


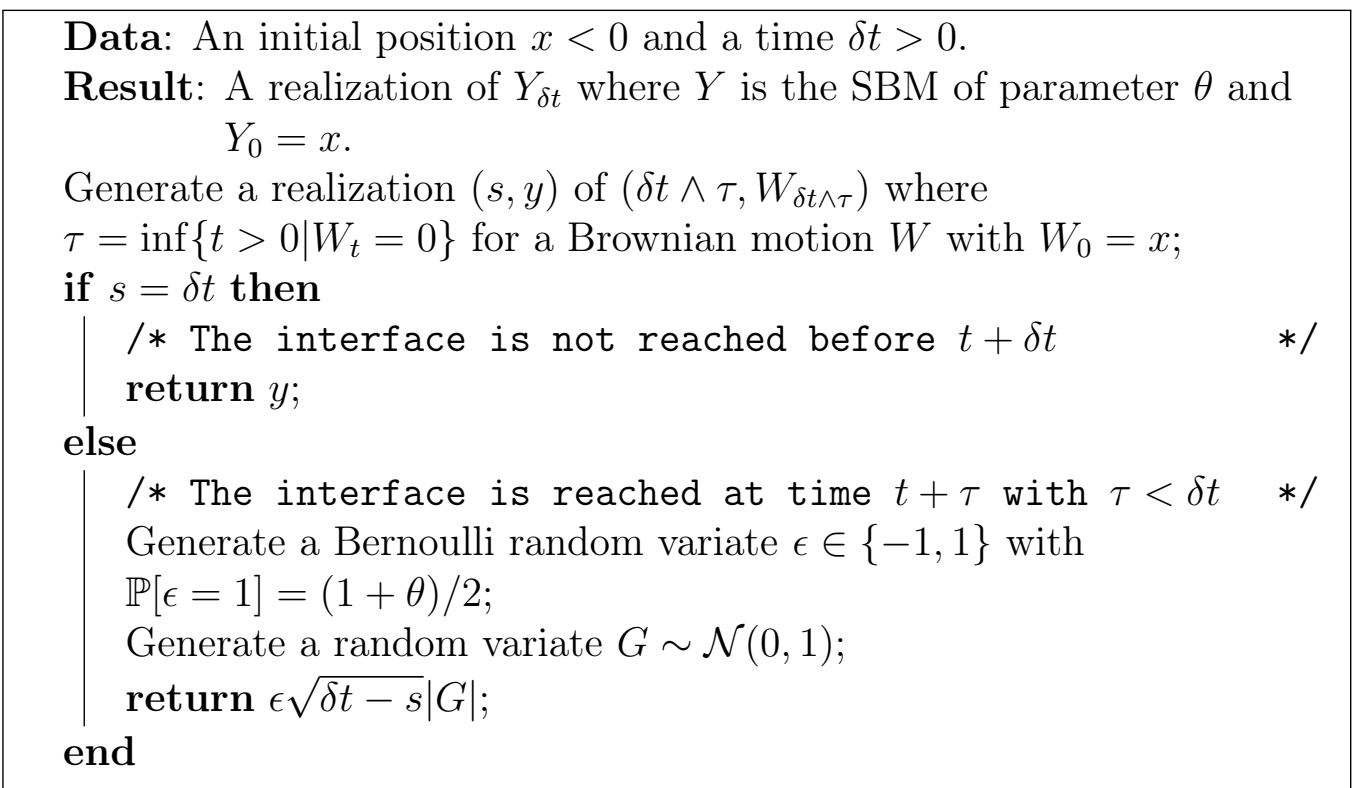

Algorithm 2: The two-steps algorithm simulating the dynamic of the SBM.

\section{A direct algorithm for the simulation of the SBM evo- lution}

We give an algorithm that does not rely on computing the first passage time to the interface, and which is then an alternative to Algorithm 2.

Now, let us consider the case where the particle is at a given point $x<$ 0 . The case $x>0$ may be considered by using the symmetry property (Property 2). In this case, one should change $x$ into $-x, y$ into $-y$ and $\theta$ into $-\theta$.

It follows from the scaling property (Property 1) that $Y_{t}$ given $\left\{Y_{t}>0\right\}$ with $Y_{0}=x$ is distributed as $\sqrt{t} Y_{1}$ given $\left\{Y_{1}>0\right\}$ with $Y_{0}=x / \sqrt{t}$.

We set

$$
z=x / \sqrt{t} \text {. }
$$

Since $\left\{Y_{1}=0\right\}$ is a null event under $\mathbb{P}_{z}$, it does not matter to consider the event $\left\{Y_{1}>0\right\}$ instead of $\left\{Y_{1} \geq 0\right\}$. With $\mathfrak{g}(y)=\mathfrak{g}(1, y)$ the density of the normal random variable $\mathcal{N}(0,1)$, the probability that $Y_{1}>0$ is

$$
\begin{aligned}
& \kappa(z)=\mathbb{P}_{z}\left[Y_{1}>0\right]=\int_{0}^{+\infty} \mathfrak{p}_{\theta}(1, z, y) d y \\
&=(1+\theta) \int_{-z}^{+\infty} \mathfrak{g}(y) d y=(1+\theta) \bar{\Phi}(-z) .
\end{aligned}
$$


With the scaling property, $\mathbb{P}_{x}\left[Y_{t}>0\right]=\kappa(z)=\kappa(x / \sqrt{t})$.

If $x>0$, then a similar computation or a symmetry argument (Property 2) shows that

$$
\mathbb{P}_{x}\left[Y_{t}>0\right]=1-(1-\theta) \bar{\Phi}(z) .
$$

Let us introduce the random variable $Z^{+}(z)$ distributed as $Y_{1}$ given $Y_{1}>0$ and $Y_{0}=z$, as well as $Z^{-}(z)$ distributed as $Y_{1}$ given $Y_{1}<0$ and $Y_{0}=z$.

Below, we study the main properties of these random variables (simulation, moments, approximations). The simulation of $Y_{\delta t}$ is then given by Algorithm 3, which is an alternative to Algorithm 2. The simulation of $Z^{+}(z)$ and $Z^{-}(z)$ are given in Algorithms 4, 5 and 6 below.

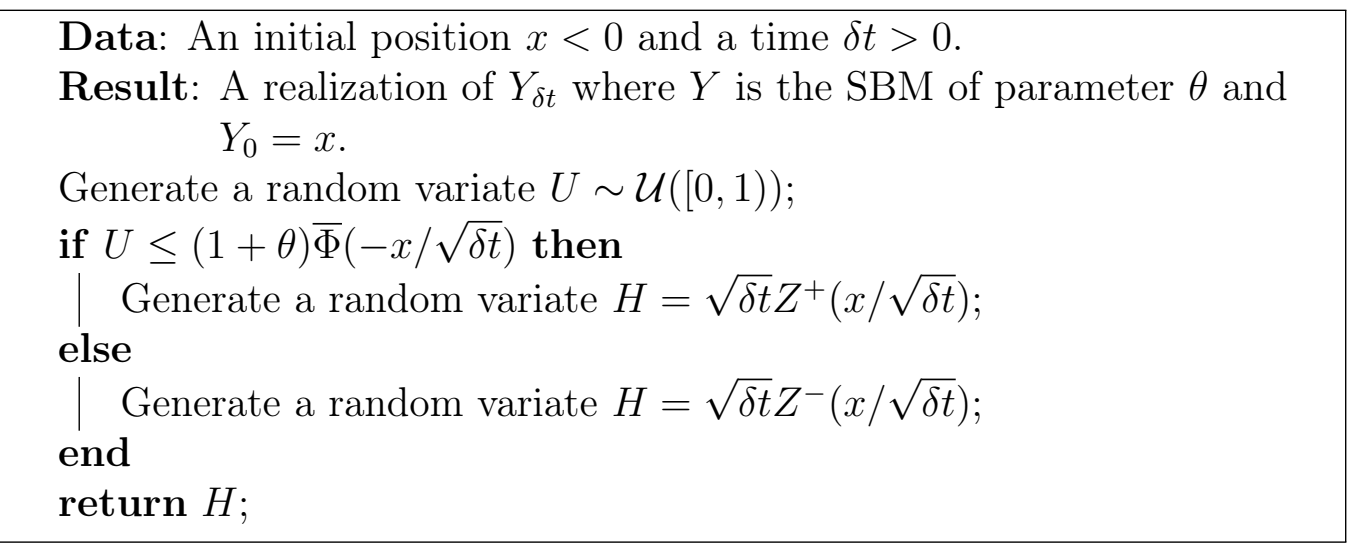

Algorithm 3: The direct algorithm simulating the dynamic of the SBM.

\subsection{Simulation of $Z^{+}$}

Assume without loss of generalities that $x<0$. With $(5), \mathfrak{p}_{\theta}(t, x, y)=$ $(1+\theta) \mathfrak{g}(y-x)$ when $y>0$ and $\kappa(x)=(1+\theta) \bar{\Phi}(-x)$.

For a bounded, measurable function $f$ with a support included in $\mathbb{R}_{+}$,

$$
\begin{aligned}
\mathbb{E}_{z}\left[f\left(Y_{1}\right) \mid Y_{1} \geq 0\right]= & \frac{\mathbb{E}_{z}\left[f\left(Y_{1}\right) ; Y_{1} \geq 0\right]}{\mathbb{P}_{z}\left[Y_{1} \geq 0\right]}=\frac{1}{\bar{\Phi}(-z)} \int_{0}^{+\infty} f(y) \mathfrak{g}(y-z) d y \\
& =\frac{1}{\bar{\Phi}(-z)} \int_{-z}^{+\infty} f(y+z) \mathfrak{g}(y) d y=\mathbb{E}[f(z+G) \mid G>-z]
\end{aligned}
$$

with $G \sim \mathcal{N}(0,1)$. This means that $Z^{+}(z)$ is distributed like $G+z$ given $\{G \geq-z\}$. The distribution of $G \sim \mathcal{N}(0,1)$ given $\{G \geq m\}$ is called the Gaussian tail distribution $\mathcal{G} \mathcal{T}(m)$.

In A, we present some ways to simulate a random variate with the Gaussian tail distribution $\mathcal{G} \mathcal{T}(z)$. Hence, the simulation of $Z^{+}(z)$ is pretty easy, as shown in Algorithm 4. 
Data: A initial position $z<0$.

Result: A random variate of $Z^{+}(z)$.

Generate a random variate $G \sim \mathcal{G} \mathcal{T}(-z)$;

return $z+G$;

Algorithm 4: Simulation of $Z^{+}(z)$ when $z<0$.

\subsection{Simulation of $Z^{-}$}

The probability that $Y_{1}<0$ with $Y_{0}=z<0$ is $1-\kappa(z)=1-(1+\theta) \bar{\Phi}(-z)$.

The distribution function of $Z^{-}(z)$ is

$$
\begin{aligned}
\mathfrak{P}^{-}(y)=\mathbb{P}_{z}\left[Z^{-}(z) \leq y\right]=\mathbb{P}_{z}\left[Y_{1} \leq y \mid Y_{1} \leq 0\right] \\
=\frac{1}{1-(1+\theta) \bar{\Phi}(-z)}(\Phi(y-z)-\theta \Phi(y+z)), y \leq 0,
\end{aligned}
$$

while its density is

$$
\mathfrak{p}_{\theta}^{-}(y)=\frac{1}{1-\kappa(z)}(\mathfrak{g}(y-z)-\theta \mathfrak{g}(y+z)), y \leq 0 .
$$

Yet $\mathfrak{g}(y-z)$ is the density of $z+G$ with $G \sim \mathcal{N}(0,1)$.

It is then possible to use a Newton algorithm in order to compute $\left(\mathfrak{P}^{-}\right)^{-1}(U)$ with $U \sim \mathcal{U}([0,1))$, which gives the desired random variable. However, this method may be time consuming.

An alternative way of proceeding is the following and depends on the sign of $\theta$.

If $\theta>0$, then one may use a rejection method. Note that

$$
\mathfrak{p}_{\theta}^{-}(y) \leq \frac{1}{1-(1+\theta) \bar{\Phi}(-z)} \mathfrak{g}(y-z) .
$$

Indeed, $\mathfrak{g}(y-z)$ is the density of $G+z$ with $G \sim \mathcal{N}(0,1)$ and $\mathbb{P}[G \leq-z]=$ $\Phi(-z)=1-\bar{\Phi}(-z)$. Hence $h(y)=\mathfrak{g}(y-z) / \Phi(-z)$ is the density of $H=z-G$ with $G \sim \mathcal{G} \mathcal{T}(z)$.

Thus,

$$
\mathfrak{p}_{\theta}^{-}(y) \leq \beta(z) h(y) \text { with } \beta(z)=\frac{1-\bar{\Phi}(-z)}{1-(1+\theta) \bar{\Phi}(-z)}
$$

A variant of the rejection techniques is the following, when one has a bound of type (6) on densities (See [18, II.3, p. 40]): Simulate a random variate $H$ with density $h$ as well as a uniform random variate $U$ until 
$\mathfrak{p}_{\theta}^{-}(H) / h(H) \leq \beta(z) U$. Then $H$ is a realization of a random variable with density $\mathfrak{p}_{\theta}^{-}$.

Note that in our case,

$$
\frac{\mathfrak{p}_{\theta}^{-}(y)}{h(y) \beta(z)}=1-\theta \frac{\mathfrak{g}(y+z)}{\mathfrak{g}(y-z)}=1-\theta \exp (-2 y z) .
$$

Since $1-U$ and $U$ have the same distribution when $U \sim \mathcal{U}([0,1))$, the stop condition in the rejection method takes a simple form.

Algorithm 5 describes the simulation of $Z^{-}(z)$ when $\theta>0, z<0$.

Data: A point $z<0$ and a parameter $\theta>0$.

Result: A random variate of $Z^{-}(z)$.

repeat

Generate a random variate $H=z-G$ with $G \sim \mathcal{G} \mathcal{T}(z)$;

Generate a random variate $U \sim \mathcal{U}([0,1))$;

until $U \geq \theta \exp (-2 H z)$;

Algorithm 5: Simulation of $Z^{-}(z)$ when $\theta>0, z<0$.

If $\theta<0$, then one may use a composition method. Let us note that

$$
\mathfrak{P}^{-}(y)=\frac{\Phi(-z)}{1-\kappa(z)} \frac{\Phi(y-z)}{\Phi(-z)}-\theta \frac{\Phi(z)}{1-\kappa(z)} \frac{\Phi(y+z)}{\Phi(z)} .
$$

The function $\Phi(y-z) / \Phi(-z)$ is the distribution function of $G+z$ given $\{G \leq-z\}$ while $\Phi(y+z) / \Phi(z)$ is the distribution function of $G-z$ given $\{G \leq z\}$.

Using the symmetry of the Gaussian distribution, $\Phi(z)=\bar{\Phi}(-z)$ for any $z \in \mathbb{R}$ and $1-\kappa(z)=\Phi(-z)-\theta \bar{\Phi}(-z)$ since $\Phi(-z)+\bar{\Phi}(-z)=1$ for any $z \in \mathbb{R}$.

For

$$
\alpha(z)=\frac{\Phi(-z)}{1-\kappa(z)}=\frac{1-\bar{\Phi}(-z)}{1-(1+\theta) \bar{\Phi}(-z)},
$$

$\mathfrak{P}^{-}(y)$ may be written

$$
\mathfrak{P}^{-}(y)=\alpha(z) \frac{\Phi(y-z)}{\Phi(-z)}+(1-\alpha(z)) \frac{\Phi(y+z)}{\Phi(z)} .
$$

One may then simulate $Z^{-}(z)$ using the composition method [18, II.4, p. 66] given by Algorithm 6 .

As previously, we are reduced to simulate Gaussian tail distributions introduced in Section 5.1 and A. 
Data: A point $z<0$ and a parameter $\theta<0$.

Result: A random variate of $Z^{-}(z)$.

Generate a uniform random variate $U \sim \mathcal{U}([0,1))$;

Compute $\alpha(z)$ given by (7);

if $U<\alpha(z)$ then

Generate $y=z-G$ with $G \sim \mathcal{G} \mathcal{T}(z)$;

else

Generate $y=-z-G$ with $G \sim \mathcal{G} \mathcal{T}(-z)$;

end

return $y$;

Algorithm 6: Simulation of $Z^{-}(z)$ when $\theta<0$.

\subsection{The moments of the random variables $Z^{+}$and $Z^{-}$}

The moments of the random variables $Z^{+}$and $Z^{-}$will be used to get some uniform or Gaussian approximations of them.

After standard computations, for $z<0$,

$$
\mathbb{E}\left[Z^{+}(z)\right]=\frac{\mathfrak{g}(z)}{\bar{\Phi}(-z)}+z, \mathbb{E}\left[Z^{-}(z)\right]=\frac{(\theta-1) \mathfrak{g}(z)}{1-(1+\theta) \bar{\Phi}(-z)}+z,
$$

and

$$
\begin{aligned}
& \mathbb{E}\left[\left(Z^{+}(z)\right)^{2}\right]=z \frac{\mathfrak{g}(z)}{\bar{\Phi}(-z)}+z^{2}+1 \\
& \mathbb{E}\left[\left(Z^{-}(z)\right)^{2}\right]=\frac{-z \mathfrak{g}(z)(1+\theta)}{1-(1+\theta) \bar{\Phi}(-z)}+1+z^{2}
\end{aligned}
$$

\subsection{Uniform distribution of $Z^{+}$and $Z^{-}$}

A first approximation consists in replacing the random variables $Z^{+}\left(\right.$resp. $Z^{-}$) by a uniform random variable $L^{+} U$ (resp. $\left.-L^{-} U\right)$ with $U \sim \mathcal{U}([0,1))$.

This means that the density $\mathfrak{p}_{\theta}^{+}$(resp. $\mathfrak{p}_{\theta}^{-}$) is replaced by the density $\left(L^{+}\right)^{-1} \mathbf{1}_{\left[0, L^{+}\right)}\left(\operatorname{resp} .\left(L^{-}\right)^{-1} \mathbf{1}_{\left[0, L^{-}\right.}\right)$.

A natural way to choose the parameter $L^{ \pm}$is by matching the means of $Z^{ \pm}$and $\pm L^{ \pm} U$. Since the expectation of $U$ is $1 / 2$, we take

$$
L^{ \pm}= \pm 2 \mathbb{E}\left[Z^{ \pm}(z)\right]
$$

\subsection{Half-normal approximation of $Z^{+}$and $Z^{-}$}

The density $\mathfrak{p}_{\theta}^{ \pm}$has a support on $\mathbb{R}_{ \pm}$. In order to get faster computations, one may want to replace $Z^{+}$(resp. $Z^{-}$) by a simpler random variable. An obvious 
choice consists in using a half-normal distribution with parameter $\sigma^{+}$. By this, we mean $|G|$ (resp. $-|G|)$, where $G \sim \mathcal{N}\left(0, \sigma^{+}\right)$(resp. $G \sim \mathcal{N}\left(0, \sigma^{-}\right)$).

The density of $|G|$ is $2 \mathfrak{g}\left(\sigma^{+}, \cdot\right)$ on $\mathbb{R}_{+}$.

The Kullback-Leibler divergence is a way to characterize the distance between two distributions. Hence, we choose the parameter $\sigma^{+}$in order to reduce the Kullback-Leibler divergence between $Z^{+}$and $|G|$ with $G \sim$ $\mathcal{N}\left(0, \sigma^{+}\right)$. This divergence is defined by

$$
\mathcal{K}\left(\mathfrak{p}_{\theta}^{+}, 2 \mathfrak{g}\left(\sigma^{+}, \cdot\right)\right)=\int_{0}^{+\infty} \mathfrak{p}_{\theta}^{+}(x) \log \frac{\mathfrak{p}_{\theta}^{+}(x)}{2 \mathfrak{g}\left(\sigma^{+}, x\right)} d x
$$

Optimizing over $\sigma^{+}$, one gets that

$$
\sigma^{+}=\int_{0}^{+\infty} x^{2} \mathfrak{p}_{\theta}^{+}(x) d x=\mathbb{E}\left[\left(Z^{+}(z)\right)^{2}\right]
$$

\section{Benchmark tests}

To show the effectiveness of our methods, we consider some test cases presented in [31] with the discontinuity at $x_{d}=0$. We consider a $1 \mathrm{D}$ domain, $[-L, L]$ with $L=49$. We have $D=D^{+}$on $[0, L]$ and $D=D^{-}$on $[-L, 0]$ with reflecting boundary conditions on $x_{\min }=-L$ and $x_{\max }=L$. The time step of the algorithm is $\delta t=0.005$.

\subsection{Test 1: density test}

On Figure 3, we plot an approximation of the density of the particles at time $T=6$ when the starting point $x_{0}$ is -5 for $D^{+}=0.5$ and $D^{-}=10, \delta t=5 \times$ $10^{-3}$, and $c_{\alpha}=3$. We use 100,000 particles and the simulation is performed using Algorithm 1 coupled with Algorithm 3 (direct SBM algorithm). The interface layer is, in that case, $\left[-\sqrt{D^{-}} c_{\alpha} \sqrt{\delta t}, \sqrt{D^{+}} c_{\alpha} \sqrt{\delta t}\right]=[-0.6708,0.15]$. At time $T=6$, the probability that a particle has reached $x_{\min }$ or $x_{\max }$ is very small. Hence, the density may be compared to the density $\mathfrak{q}(t, x, y)$ on the whole space and we see a perfect match. We obtain the same good result by using the two-steps Algorithm 2 instead of Algorithm 3 (not drawn).

Remark 5. In order to compare these expression with the analytic expressions found for example in [31,43], one has to take into account the one-half factor so in these articles, correspond to $D_{2}=D^{+} / 2$ and $D_{1}=D^{-} / 2$.

\subsection{Test 2: long time behavior}

We also study the long time behavior of the scheme, as in [31]. It is known that in the steady state regime, the particles are uniformly spread over the domain, whatever the values of the diffusivity coefficient $D$. If $N^{+}(t)$ is the 


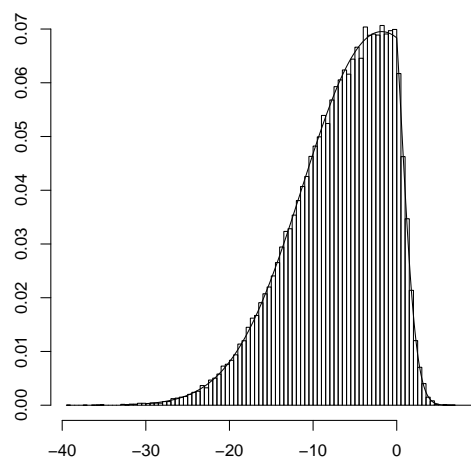

Figure 3: Normalized histogram of particles at time $T=6$ obtained with Algorithm 1 coupled with Algorithm 3 (direct SBM algorithm), with 100,000 particles when $x_{0}=-5$ for $D^{-}=10, D^{+}=0.5, \delta t=5 \times 10^{-3}$, and $c_{\alpha}=3$ in the definition of the interface layer. The black line is the density $\mathfrak{q}(t, x, y)$.

number of particles on $[0, L]$ and $N^{-}(t)$ is the number of particles on $[-L, 0]$ at time $t$, then $N^{-}(t) / N^{+}(t)$ converges to 1 . Figure 4 presents the evolution of $R(t)=N^{-}(t) / N^{+}(t)$ for $D^{-}=5$ and $D^{+}=0.25$ for 10,000 particles starting at the position $x_{\min }=-L$ with Algorithm 1 coupled with Algorithm 3 or 2 . The curve "No correction" corresponds to the result obtained with the use of a classical Euler scheme on the entire domain, even in the interface layer. It emphasizes the need of a specific algorithm inside the interface layer, like one of the two proposed here, to take the discontinuity into account.

On Figure 4, the horizontal red dashed lines represent the 99\%-confidence interval on $R(t)$ based on the quantiles of $4 Z$ and the above approximation. Indeed, by the Central Limit Theorem, assuming that the steady state is reached, then $\mathbb{P}\left[X_{t} \in[0, L]\right]=1 / 2$ and then $N^{+}(t)$ may be approximated by $N^{+}(t)=N(1 / 2+Z(t))$ where $Z(t) \sim \mathcal{N}(0,1 / 4 N)$, Hence,

$$
R(t)=\frac{\frac{1}{2}+Z}{\frac{1}{2}-Z} \approx 1+4 Z .
$$

Hence, for $t$ large enough, $R(t)$ fluctuates around 1 but these fluctuations stay within the error range of the Monte-Carlo method.

We also check the convergence of the ratio $R(t)$ towards 1 for different ratio $D^{-} / D^{+}, D^{-} / D^{+} \in\{2.5,5,7.5,10,12.5,15,17.5,20\}$. Figure 5 shows the ratio of $N^{-} / N^{+}$averaged over the time 20000-30000, for which the steady state is reached. Without any appropriate method to take the discontinuity into account, the results are not satisfactory as attested by the curve named "No correction". On the contrary, all simulations performed using one 


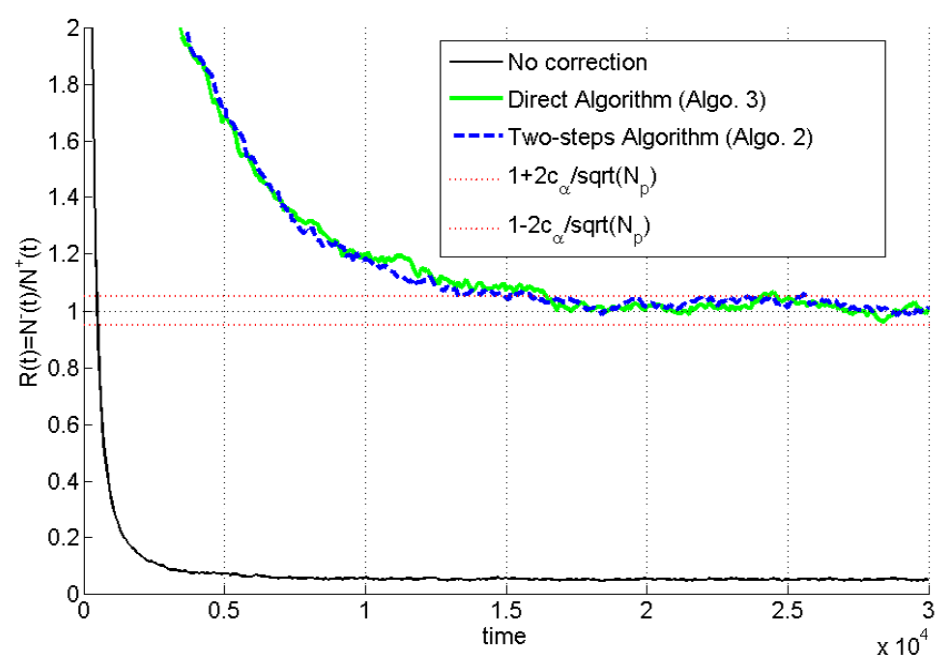

Figure 4: Convergence of the ratio $R(t)=N^{-}(t) / N^{+}(t)$ vs time with the two proposed algorithms for $D^{-} / D^{+}=20$ and 10,000 particles starting at the position $x_{\min }=-L$. The black curve "No correction" corresponds to the result obtained with the use of a classical Euler scheme on the entire domain, even in the interface layer.

of our two algorithms lead to consistent results whatever the value of the ratio $D^{-} / D^{+}$.

\section{Summary and future work}

We have proposed two effective algorithms for the simulation of the SBM dynamic. These algorithms are exact ones and are essential tools for the Lagrangian simulation of diffusion in discontinuous media. Extensive test cases as well as comparison with other algorithms (like the ones proposed in geosciences $[27,62])$ will be provided in a forthcoming paper.

Another interesting future work will be to introduce immobile times in order to consider more complex particle fates such as absorption, stagnation and desorption $[6,25,58]$.

\section{Acknowledgments}

We sincerely wish to thank the Reviewers for their fruitful comments.

\section{A Simulation of the Gaussian tail distribution}

The Gaussian tail distribution $\mathcal{G} \mathcal{T}(z)$ is the distribution of the normal random variable $G$ given to $\{G \geq z\}$. If $z<0$, then a simple rejection technique may be used: one draws a normal random variate until it is greater that $z$. 


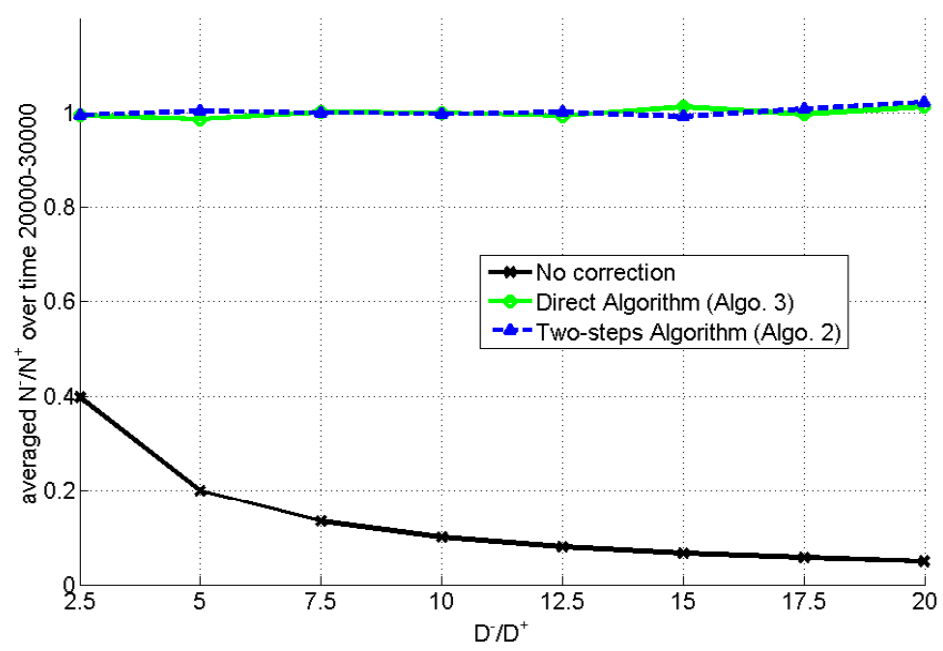

Figure 5: Convergence of the averaged ratio of $N^{-} / N^{+}$over time 20000-30000 with the two proposed algorithms for $D^{-} / D^{+} \in$ $\{2.5,5,7.5,10,12.5,15,17.5,20\}$ and 10,000 particles starting at the position $x_{\text {min }}=-L$. The black curve "No correction" corresponds to the result obtained with the use of a classical Euler scheme on the entire domain, even in the interface layer.

With $z=0$, it takes two samples in average. When $z$ is positive, this technique becomes inefficient as $z$ increases. A specific rejection technique given in [18, IX.1, p. 380] provides an efficient way to sample $G$ given $\{G \geq z\}$ for $z \geq 0$.

\section{B Simulation of the first time the particle reaches the interface}

We consider in this appendix how to simulate the process $X$ until the (random) time $\tau$ at which it reaches the discontinuity at 0 for the first time. Until this time $\tau$, then for a Brownian motion $X_{t}=\sqrt{D^{+}} W_{t}$ (resp. $\left.X_{t}=\sqrt{D^{-}} W_{t}\right)$ if $X_{0}>0$ (resp. $\left.X_{0}<0\right)$. The problem then reduces to the simulation of the first time the Brownian motion reaches 0 .

Given $\delta t$ and $X_{k}$, we then present several ways to get either $X_{k+1}$ with $X_{k+1} X_{k}>0$ (the two successive positions are on the same side of the discontinuity) or the first time $\tau$ the process hits 0 when starting from $X_{k}$ (or equivalently, the Brownian motion $W$ hits 0 with starting point $\left.X_{k} / \sqrt{D\left(X_{k}\right)}\right)$ with $\tau<\delta t$.

Three methods are presented here. Other algorithms have been proposed 
to perform such simulations. But some of them (See [24] for example) show their relevance in term of weak error, that is the error induced in functionals of type $\mathbb{E}\left[\phi\left(\tau, X_{\tau}\right)\right]$ where the first hitting time $\tau$ of 0 is replaced by its approximation. Other methods exist where the time step is reduced (See for example [29], which also use the Brownian bridge test in a way similar to the one presented in B.2 but with exponential time steps instead of constant time steps).

What is said here may be applied to more general diffusion processes. Using the scale, we work on the level of the Brownian motion which we may assume to start from $x$. Again, the results of Sect. 2.2 may be applied there. If $x$ is far enough from 0 , then the probability the particle crosses 0 before $\delta t$ may be neglected.

\section{B.1 Linear approximation}

We perform an Euler scheme for the Brownian motion with diffusivity $D^{+}$ or with diffusivity $D^{-}$. If the interface is crossed, the new position does not have the same sign as the starting position. This new position is kept to perform a linear approximation of $\tau$ and the new position is set to be 0 as described by Algorithm 7.

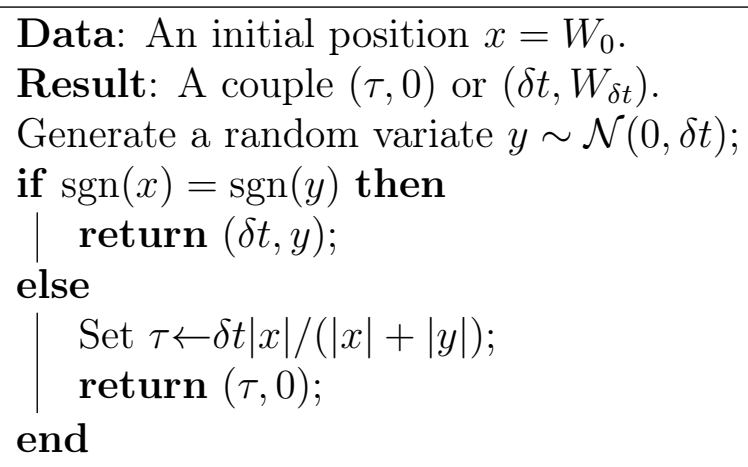

Algorithm 7: Linear approximation of $\tau$

The choice of $\tau$ in case of a crossing is justified by Remark 6 below in B.2.

The drawback with this algorithm is to over-estimate the first hitting time of 0: if $X_{k}$ and $X_{k+1}$ have the same sign, it is possible that the part of the path between times $t_{k}$ and $t_{k+1}$ crosses the boundary 0 . We refine this method in the next section.

\section{B.2 Using Brownian bridge's properties}

A way to modify the naive previous scheme consists in using the Brownian bridge distribution (see Algorithm 8). Indeed, the Brownian path $\left(W_{t}\right)_{t \in[0, \delta t]}$ 
when $X_{0}=x$ and $X_{\delta t}=y$ is called a Brownian bridge. As $\delta t$ goes to zero, the deviation of a Brownian bridge path from the segment linking $x$ to $y$ becomes smaller and smaller.

The idea is then to use its property to simulate the first time the Brownian bridge crosses 0 , if it does. For this method, see [4,10,23,40], ...

There are two cases to consider: $x y>0$ and $x y<0$. Without loss of generalities, we assume that $x<0$. The computations are borrowed from [8].

The case $x<0, y<0$. Here, the process may have not crossed the boundary.

The probability that the Brownian bridge goes above a level $\beta>y>x$ is

$$
\mathbb{P}_{x}\left[\sup _{t \in[0, \delta t]} W_{t} \geq \beta \mid W_{\delta t}=y\right]=\exp \left(\frac{-2}{\delta t}(\beta-x)(\beta-y)\right) .
$$

If the Brownian bridge crosses 0 , then the first time $\tau=\delta t \xi /(1+\xi)$ where $\xi$ follows an inverse Gaussian distribution $\mathcal{I} \mathcal{G}\left(x / y, x^{2} / \delta t\right)$. The density $r(\mu, \lambda, \cdot)$ of $\mathcal{I} \mathcal{G}(\mu, \lambda)$ is

$$
r(\mu, \lambda, x)=\sqrt{\frac{\lambda}{2 \pi x^{3}}} \exp \left(\frac{-\lambda(x-\mu)^{2}}{2 \mu^{2} x}\right) .
$$

This distribution is easily simulated using the algorithm [50] (See also [18, p. 148]).

The case $x<0, y>0$. In this case, the process crosses the boundary. Its first hitting time $\tau$ is equal to $\delta t \xi /(1+\xi)$ where $\xi$ follows an inverse Gaussian distribution $\mathcal{I} \mathcal{G}\left(-x / y, x^{2} / \delta t\right)$.

Remark 6. The mean of the $\mathcal{I G}(\mu, \lambda)$ is $\mu$ and its variance is $\mu^{3} / \lambda$. Thus, for $\xi \sim \mathcal{I} \mathcal{G}\left(-x / y, x^{2} / \delta t\right)$ with $x<0$ and $y>0$,

$$
\xi=\frac{|x|}{|y|}+\sqrt{\delta t} \alpha
$$

where $\alpha$ is a random variable with zero mean and variance $|x| /|y|^{3}$. Hence,

$$
\frac{\tau}{\delta t}=\frac{\frac{|x|}{|y|}+\sqrt{\delta t} \alpha}{1+\frac{|x|}{|y|}+\sqrt{\delta t} \alpha}=\frac{|x|+\sqrt{\delta t} \alpha^{\prime}}{|x|+|y|+\sqrt{\delta t} \alpha^{\prime}} \approx \frac{|x|}{|x|+|y|}+\sqrt{\delta t} \alpha^{\prime} \frac{|y|}{|x|(|x|+|y|)}+\cdots
$$

with $\alpha^{\prime}=\alpha|y|$ has a variance $|x| /|y|$.

Hence, for small values of $\delta t$, the random variable $\tau$ may be approximated by $\delta t|x| /(|x|+|y|)$. 
Data: An initial point $x$ and a time $\delta t>0$.

Result: A random variate $\left(\delta t \wedge \tau, W_{\delta t \wedge \tau}\right)$.

Generate a random variate $G \sim \mathcal{N}(0,1)$;

Set $y \leftarrow x+\sqrt{\delta t} G$;

if $\operatorname{sgn}(x) \neq \operatorname{sgn}(y)$ then

Generate a random variate $\xi \sim \mathcal{I} \mathcal{G}\left(|x| /|y|, x^{2} / \delta t\right)$;

Set $\tau \leftarrow \delta t \xi /(1+\xi)$;

else

return $(\tau, 0)$;

Generate a random variate $U \sim \mathcal{U}([0,1))$;

if $U<\exp (-2 x y / \delta t)$ then

Generate a random variate $\xi \sim \mathcal{I} \mathcal{G}\left(|x| /|y|, x^{2} / \delta t\right) ;$

Set $\tau \leftarrow \delta t \xi /(1+\xi)$

return $(\tau, 0)$;

else

return $(\delta t, y)$

end

end

Algorithm 8: Simulation of $\left(\delta t \wedge \tau, W_{\delta t \wedge \tau}\right)$ using Brownian bridge's properties. 


\section{B.3 Controlling exit times from intervals}

If the particle is initially at position $y$, then let us fix $x<y<z$, and let $\tau=\inf \left\{t>0 \mid W_{t} \notin[x, z]\right\}$ be the first time the Brownian motion $W$ reaches either $x$ or $z$.

The couple $\left(\tau \wedge \delta t, W_{\tau \wedge \delta t}\right)$ may be simulated exactly for any $\delta t>0[37,39]$. A C library is available for this purpose [36].

If the interface is at $z=0$ and $y<0$, then this algorithm may return $\left(\tau, X_{\tau}\right)$ with $\tau<\delta t$ and $X_{\tau}=x$. It is then possible to iterate by replacing $\delta t$ by $\delta t-\tau$ until the particle reaches the interface or the sum of the time is equal to $\delta t$, as seen in Algorithm 9.

Data: $W_{0}=y$.

Result: The hitting time $\tau$ of 0 if smaller than $\delta t$ or the next position of the Euler scheme.

Set $s \leftarrow 0$;

\section{repeat}

Select $x<y<z$ with $z=0$ if $y<0$ and $x=0$ if $y>0$;

Generate a random variate $\left(\tau^{\prime}, y^{\prime}\right)$ of $\left(\tau \wedge(\delta t-s), W_{\tau \wedge(\delta t-s)}\right)$ with $\tau=\inf \left\{t>0 \mid W_{t} \notin[x, z]\right\}$ and $W_{0}=y$ for a Brownian motion $W$;

Set $s \leftarrow s+\tau^{\prime}$;

Set $y \leftarrow y^{\prime}$;

until $y=0$ or $s=\delta t$;

Algorithm 9: Simulation of $\left(\tau \wedge \delta t, W_{\tau \wedge \delta t}\right)$ using the exit time from intervals.

Algorithm 9 is more costly than Algorithm 8. Yet this algorithm is suitable in the case of multiple interfaces, since this algorithm ensures that before $\delta t \wedge \tau$, the particle does not leave the interval $[x, z]$.

For a multi-dimensional generalizations of these simulations, using squares, rectangles or spheres, see $[12,14,15,51]$.

\section{References}

[1] P. Ackerer and R. Mose, Comment on "Diffusion theory for transport in porous media: Transition-probability densities of diffusion processes corresponding to advectiondispersion equations" by Eric M. LaBolle et al., Water Resour. Res. 36 (2000), no. 3, 819-821.

[2] T.A. Appuhamillage, V.A. Bokil, E. Thomann, E. Waymire, and B. D. Wood, Solute transport across an interface: A Fickian theory for skewness in breakthrough curves, Water Resour. Res., 46 (2010), W07511, DOI 10.1029/2009WR008258. 
[3] - Occupation and local times for Skew Brownian motion with application to dispersion accross an interface, Ann. Appl. Probab. 21 (2011), no. 1, 183-214, DOI 10.1214/10-AAP691.

[4] P. Baldi, Exact asymptotics for the probability of exit from a domain and applications to simulation, Ann. Probab. 23 (1995), no. 4, 1644-1670.

[5] M. Bechtold, J. Vanderborght, O. Ippisch, and H.V. Vereecken, Efficient random walk particle tracking algorithm for advective-dispersive transport in media with discontinuous dispersion coefficients and water contents, Water Resour. Res., 47 (2011), W10526.

[6] D.A. Benson and M.M. Meerschaert, A simple and efficient random walk solution of multi-rate mobile/immobile mass transport equations, Advances in Water Resources 32 (2009), 532-539.

[7] B. Berkowitz, A. Cortis, I. Dror, and H. Scher, Laboratory experiments on dispersive transport across interfaces: The role of flow direction, Water Resour. Res., 45 (2009), no. W02201.

[8] A Beskos and G.O. Roberts, Exact simulation of diffusion, Ann. Appl. Probab. 15 (2005), no. 4, 2422-2444, DOI 10.1214/105051605000000485.

[9] M. Bossy, N. Champagnat, S. Maire, and D. Talay, Probabilistic interpretation and random walk on spheres algorithms for the Poisson-Boltzmann equation in Molecular Dynamics, ESAIM M2AN 44 (2010), no. 5, 997-1048.

[10] F. Buchman, Simulation of stopped diffusion, J. Comput. Phys. 202 (2005), no. 2, 446-462, DOI 10.1016/j.jcp.2004.07.009.

[11] A. Cortis and A. Zoia, Model of dispersive transport across sharp interfaces between porous materials, Phys. Rev. E 80 (2009), 011122.

[12] F. Campillo and A. Lejay, A Monte Carlo method without grid for a fractured porous domain model, Monte Carlo Methods Appl. 8 (2002), no. 2, 129-148.

[13] R.S. Cantrell and C. Cosner, Diffusion Models for Population Dynamics Incorporating Individual Behavior at Boundaries: Applications to Refuge Design, Theor. Popul. Biol. 55 (1999), no. 2, 189-207, DOI 10.1006/tpbi.1998.1397.

[14] M. Deaconu and S. Herrmann, Hitting time for Bessel processes - walk on moving spheres algorithm (2011), available at arxiv: :1111.3736. Preprint.

[15] M. Deaconu and A. Lejay, A random walk on rectangles algorithms, Methodol. Comput. Appl. Probab. 8 (2006), no. 1, 135-151, DOI 10.1007/s11009-006-7292-3.

[16] M. Decamps, M. Goovaerts, and W. Schoutens, Self exciting threshold interest rates models, International Journal of Theoretical and Applied Finance 9 (2006), no. 7, 1093-1122, DOI 10.1142/S0219024906003937.

[17] F. Delay, Ph. Ackerer, and C. Danquigny, Simulating Solute Transport in Porous or Fractured Formations Using Random Walks Particle Tracking: A Review, Vadose Zone J. 4 (2005), 360-379.

[18] L. Devroye, Non-Uniform Random Variate Generation, Springer-Verlag, 1986.

[19] P. Étoré, On random walk simulation of one-dimensional diffusion processes with discontinuous coefficients, Electron. J. Probab. 11 (2006), no. 9, 249-275. 
[20] P. Étoré and A. Lejay, A Donsker theorem to simulate one-dimensional processes with measurable coefficients, ESAIM Probab. Stat. 11 (2007), 301-326, DOI $10.1051 / \mathrm{ps}: 2007021$.

[21] P. Étoré and M. Martinez, Exact simulation of one-dimensional stochastic differential equations involving the local time at zero of the unknown process (2011), available at arXiv:1102.2565. Preprint.

[22] E. Fieremans, D. S Novikov, J. H. Jensen, and J. A. Helpern, Monte Carlo study of a two-compartment exchange model of diffusion, NMR Biomed. 23 (2010), 711-724, DOI 10.1002/nbm.1577.

[23] E. Gobet, Weak approximation of killed diffusion using Euler schemes, Stochastic Process. Appl. 87 (2000), no. 2, 167-197, DOI 10.1016/S0304-4149(99)00109-X.

[24] E. Gobet and S. Menozzi, Stopped diffusion processes: boundary corrections and overshoot, Stochastic Process. Appl. 120 (2010), no. 2, 130-162, DOI 10.1016/j.spa.2009.09.014.

[25] R. Haggerty and S. M. Gorelick, Multiple-Rate Mass Transfer for Modeling Diffusion and Surface Reactions in Media with Pore-Scale Heterogeneity, Water Resour. Res. 31 (1995), no. 10, 2383-2400, DOI 10.1029/95WR10583.

[26] J. M. Harrison and L. A. Shepp, On skew Brownian motion, Ann. Probab. 9 (1981), no. $2,309-313$.

[27] H. Hoteit, R. Mose, A. Younes, F. Lehmann, and Ph. Ackerer, Three-dimensional modeling of mass transfer in porous media using the mixed hybrid finite elements and the random-walk methods, Math. Geology 34 (2002), no. 4, 435-456.

[28] K. Itô and H.P. McKean, Diffusion processes and their sample paths, 2nd ed., SpringerVerlag, 1974.

[29] K. M. Jansons and G. D. Lythe, Exponential timestepping with boundary test for stochastic differential equations, SIAM J. Sci. Comput. 24 (2003), no. 5, 1809-1822, DOI 10.1137/S1064827501399535.

[30] I. Karatzas and S. E. Shreve, Brownian motion and stochastic calculus, 2nd ed., Graduate Texts in Mathematics, vol. 113, Springer-Verlag, New York, 1991.

[31] E. M. LaBolle, G.E. Fogg, and A.F.B. Thomson, Random-Walk Simulation of Transport in Heterogeneous Porous Media: Local Mass-Conservation Problem and Implementation Methods, Water Resour. Res., 32 (1996), no. 3, 582-593, DOI 10.1029/95WR03528.

[32] E. M. LaBolle and Y. Zhang, Reply to comment by D.-H. Lim on "Diffusion processes in composite porous media and their numerical integration by random walks: Generalized stochastic differential equations with discontinuous coefficients", Water Resour. Res. 42 (2006), W02602, DOI 10.1029/2005WR004403.

[33] E. M. LaBolle, J. Quastel, G. E. Fogg, and J. Gravner, Diffusion processes in composite porous media and their numerical integration by random walks: Generalized stochastic differential equations with discontinuous coefficients, Water Resour. Res. 36 (2000), 651-662, DOI 10.1029/1999WR900224.

[34] A. Lejay, Simulation of a stochastic process in a discontinuous layered medium, Electron. Comm. Probab. 16 (2011), 764-774. 
[35] O. A. Ladyženskaja, V. Ja. Rivkind, and N. N. Ural'ceva, Solvability of diffraction problems in the classical sense, Trudy Mat. Inst. Steklov. 92 (1966), 116-146.

[36] A. Lejay, exitbm: a library for simulating exit times and positions for the Brownian motion from simple domains, Technical Report RT-0402, INRIA, 2011. Description of a C library, downloadable at exitbm.gforge.inria.fr.

[37] _ Simulating a diffusion on a graph. Application to reservoir engineering, Monte Carlo Methods Appl. 9 (2003), no. 3, 241-256.

[38] _ On the constructions of the Skew Brownian motion, Probab. Surv. 3 (2006), $413-466$.

[39] A. Lejay and M. Martinez, A scheme for simulating one-dimensional diffusion processes with discontinuous coefficients, Ann. Appl. Probab. 16 (2006), no. 1, 107-139, DOI 10.1214/105051605000000656.

[40] H. R. Lerche and D. Siegmund, Approximate exit probabilities for a Brownian bridge on a short time interval, and applications, Adv. in Appl. Probab. 21 (1989), no. 1, $1-19$, DOI 10.2307/1427195.

[41] D.-H. Lim, Comment on "Diffusion processes in composite porous media and their numerical integration by random walks: Generalized stochastic differential equations with discontinuous coefficients" by E. M. LaBolle, J. Quastel, G. E. Fogg, and J. Gravner, Water Resour. Res. 42 (2006), W02601, DOI 10.1029/2005WR004091.

[42] A. Marcowith and F. Casse, Postshock turbulence and diffusive shock acceleration in young supernova remnants, Astron. Astrophys. 515 (2010), no. A90, DOI 10.1051/0004-6361/200913022.

[43] M. Marseguerra and A. Zoia, Normal and anomalous transport across an interface: Monte Carlo and analytical approach, Ann. Nucl. Energy 33 (2006), no. 17-18, 13961407, DOI 10.1016/j.anucene.2006.09.012.

[44] M. Martinez, Interprétations probabilistes d'opérateurs sous forme divergence et analyse de méthodes numériques associées, Université de Provence / INRIA SophiaAntipolis, 2004.

[45] M. Martinez and D. Talay, Discrétisation d'équations différentielles stochastiques unidimensionnelles à générateur sous forme divergence avec coefficient discontinu, C. R. Math. Acad. Sci. Paris 342 (2006), no. 1, 51-56, DOI 10.1016/j.crma.2005.10.025.

[46] __ One-Dimensional parabolic diffraction equations: Pointwise estimates and discretization of related stochastic differential equations with weighted local times (2011). Preprint.

[47] M. Mascagni and N. A. Simonov, Monte Carlo methods for calculating some physical properties of large molecules, SIAM J. Sci. Comput. 26 (2004), no. 1, 339-357, DOI $10.1137 /$ S1064827503422221.

[48] R. Metzler and J. Klafter, The restaurant at the end of the random walk: recent developments in the description of anomalous transport by Fractional Dynamics., J. Phys. A: Math. Gen. 37 (2004), R161-208.

[49] _ The random walk's guide to anomalous diffusion: a Fractional Dynamics approach., Phys. Rep. 339 (2000), 1-77. 
[50] J.R. Michael, W.R. Shucany, and R.W. Haas, Generating random variates using transformations with multiple roots, American Statistician 30 (1976), no. 2, 88-90.

[51] G.N. Milstein and M.V. Tretyakov, Simulation of a space-time bounded diffusion, Ann. Appl. Probab. 9 (1999), no. 3, 732-779.

[52] Montroll E. W. and Weiss G. H., Random walks on lattices. II., J. Math. Phys. 6 (1965), 167-181.

[53] O. Ovaskainen and S. J. Cornell, Biased movement at a boundary and conditional occupancy times for diffusion processes, J. Appl. Probab. 40 (2003), no. 3, 557-580.

[54] J.M. Ramirez, Skew Brownian Motion and Branching Processes Applied to DiffusionAdvection in Heterogenous Media and Fluid Flow, Ph.D. thesis, Oregon State University, 2007.

[55] Jorge M. Ramirez, Enrique A. Thomann, Edward C. Waymire, Roy Haggerty, and Brian Wood, A generalized Taylor-Aris formula and skew diffusion, Multiscale Model. Simul. 5 (2006), no. 3, 786-801, DOI 10.1137/050642770.

[56] J. Ramirez, E. Thomann, E. Waymire, J. Chastenet, and B. Wood, A Note on the Theoretical Foundations of Particle Tracking Methods in Heterogeneous Porous Media, Water Resour. Res. 44 (2007), W01501, DOI 10.1029/2007WR005914.

[57] P. Salamon, D. Fernandez-Garcia, and J. J. Gomez-Hernandez, A review and numerical assessment of the random walk particle tracking method, J. Contaminant Hydrology 87 (2006), no. 3-4, 277-305, DOI 10.1016/j.jconhyd.2006.05.005.

[58] P. Salamon, D. Fernàndez-Garcia, and J. J. Gómez-Hernández, Modeling mass transfer processes using random walk particle tracking, Water Resour. Res. 42 (2006), no. W11417, DOI 10.1029/2006WR004927.

[59] K. Semra, Modélisation tridimensionnel le du transport d'un traceur en milieux poreux saturé: évaluation des théories stochastiques, Thèse de doctorat, Université Louis Pasteur, Strasbourg, 1994.

[60] D. W. Stroock, Diffusion semigroups corresponding to uniformly elliptic divergence form operators, Séminaire de Probabilités, XXII, Lecture Notes in Math., vol. 1321, Springer, Berlin, 1988, pp. 316-347.

[61] D.J. Thomson, W.L. Physick, and R.H. Maryon, Treatment of Interfaces in Random Walk Dispersion Models, J. Appl. Meteorol. 36 (1997), 1284-1295.

[62] G.J.M. Uffink, A random walk method for the simulation of macrodispersion in a stratified aquifer, Relation of Groundwater Quantity and Quality, IAHS Publication (IAHS Publication, ed.), IAHS Press, Walingford, UK, 1985, pp. 103-114.

[63] J.B. Walsh, A diffusion with discontinuous local time, Temps locaux, Astérisques, Société Mathématique de France, 1978, pp. 37-45.

[64] M. Zhang, Calculation of diffusive shock acceleration of charged particles by skew Brownian motion, Astrophys. J. 541 (2000), 428-435. 